REVISIÓN DE TEMA
Recibido: $21 / 05 / 2019$

Revisado: 21/05/2019

Aprobado: 08/06/2019

\title{
Muros A derruir. \\ A propósito de fuentes de datos y método histórico
}

\author{
Walls to pull down. \\ In relation to data sources and historical method
}

\author{
Vicente Fernando Salas Salazar \\ Sociólogo, Universidad de Nariño. \\ Magíster Sociología, Universidad Nacional de Colombia - Sede Bogotá. \\ Master universitario en Antropología Filosófica \\ Doctorando en Estudios Territoriales, Universidad Autónoma de Tlaxcala - México D.F. \\ Email:vsalas_salazar@hotmail.com
}

Escribo sobre todo para conversar, para mostrarme al otro y descubrirlo, ese otro que me define y sin el cual soy un animal sin casa, una voz inaudible en el vacío. Sin embargo, sé que las palabras mienten, enredan, son luz que convierte la realidad en un baile de sombras. El lenguaje revela, descubre, disuelve la grisalla, después, él mismo se transforma en velo y

oculta el universo de lo no nombrado, de lo que se reprime y olvida, de todo lo que vive en el silencio. Redacto unas notas y las dirijo a ti, o a él, al eco que teje una malla con las voces (De la Torre, 1999, p. 1).

Doi: https://doi.org/10.22267/rceilat.194445.26

\begin{abstract}
Resumen
Apuesto a que esta idea de resumen funja como una idea provocadora; para lograr tal propósito, asumo la postura de revelar un conjunto de enunciados que no están expuestos de manera explícita en el texto, que no se encuentran en el texto pero que si se recrean de manera permanente como intertextualidad. Lo interesante es, y, de entrada; revelar lo "no dicho" como texto en la idea de resumen, situar al sujeto en la ruta y anticipar el momento hermenéutico.

Podría decir, que estos tiempos; los que nosotros estamos viviendo y los lenguajes con los cuales los estamos leyendo y comprendiendo, son propios para el reconocimiento de un mundo en constante dispersión y asociacionismo libre, de ambivalencia e incesante invención, de la presencia de lo alternativo y lo colateral, de la correlación de cosas e ideas de particular ambigüedad y oscilación, del lenguaje denotativo que se ha vuelto arte musical Jazz y que se arma a través de un compás
\end{abstract}


de cadencias mediado por la presencia de ritmos abiertos, del ritmo que libera al lenguaje de la fría gramática, de lo liso y lo "solido" y lo devuelve; como en un principio, esponja y simultaneidad porosa. Bien podría decirse entonces que el "combate" de la vida contemporánea se está dando en estas dimensiones. ¿Estamos asistiendo acaso al desmoronamiento de los territorios compactos, "cerrados" por la creación de espacios indeterminados, "abiertos"?. Lo que se deja entrever entonces es la presencia de una nueva manera de asirse en el mundo. Decir entonces que nuestra vida en el mundo de hoy se expresa de manera abierta, lisa y nómada; modo rizomático diría Gillez Deleuze, es una lectura de aproximación más "subversiva" de los "grandes" paradigmas que dominaron la lógica del mundo occidental desde los tiempos de Aristóteles, que se proyectaron como los saberes de la verdad y que hoy, gracias a las nuevas líneas de fuga que abre esta sana y necesaria "subversión" se aperturan nuevas y novedosas formas de indagar estas realidades sociológicas, lejos del fantasma de la verdad.

Teniendo como suelo patrio este precepto, me moveré hacia el complejo universo de la llamada "investigación social" y el dilema que apertura el modo rizomático, de esta nueva realidad intersticial a la que debemos enfrentamos los investigadores cuando adelantamos nuestra tarea interrogativa. ¿ puede traducirse en un obstáculo serio si no se pone en discusión el asunto del método?, salvar este obstáculo puede ser el mejor comienzo para iluminar la ruta de investigación y el investigador social podrá hacer de su práctica un ejercicio de buen aliento.

No obstante, vale la pena considerar un elemento central y que hace la diferencia respecto al modo y a manera en que nos enfrentamos y leemos estas nuevas realidades, el de la teoría. Son muy frecuentes las polémicas acerca del papel que debe cumplir la teoría y la relevancia que habrá de atribuirle el investigador. Al respecto tengo que decir que la teoría es el elemento que reviste de importancia el proceso de investigación. Es decir, es en la práctica de la investigación donde se vuelve operativa la teoría y esto es lo que le da sentido al oficio de la investigación social. Esto debe traducirse en una suerte de ejercicio "de fenomenología hermenéutica"

Palabras clave: Método; Historia; Hermenéutica; Investigación

\begin{abstract}
I bet this idea of abstract serve as a provocative idea; to achieve that purpose, I assume position reveal a set of statements that are not exposed explicitly in the text, which are not found in the text but if you recreate permanently as Intertextuality. The interesting thing is, and input; "” reveal the "unspoken" as text in the idea of summary, put the subject on the route and anticipate the hermeneutic moment.

You might say, that these times; which we are living and the languages with which them we are reading and understanding, are themselves for the recognition of a world in constant dispersion and free associations, ambivalence and ceaseless invention, of the presence of alternative and as collateral, the correlation of things
\end{abstract}


and ideas of particular ambiguity and swing, the denotative language which has become a Jazz musical art and arms through a compass of cadences mediated by the presence of open rhythms, rhythm that frees the language of the cold, flatness and grammar as "solid" and returns it; Initially, sponge and porous concurrency. Well it could be said that the "battle" of contemporary life is taken in these dimensions. Are we witnessing to the collapse of the compact Territories, "closed" by the creation of indeterminate, "open" spaces? What be glimpsed then is the presence of a new way of grasping the world. Say that our life in today's world is expressed openly, smooth and nomadic; Rhizomatic way would say Gillez Deleuze, is a reading of approach more "subversive" of the "big" paradigms that dominated the logic of the Western world since the time of Aristotle, who were projected as the knowledge of the truth and now, thanks to the new lines of flight that opens this healthy and necessary 'subversion' will span new and innovative ways to investigate these sociological realities, away from the ghost of the truth.

View homeland to this precept, I will move me towards the complex universe of the so-called "social research" and the dilemma that opening the Rhizomatic way, of this new interstitial reality which we must we face researchers when we anticipate our question task. It can result in a serious obstacle if not put in discussion the issue of the method?, save this obstacle may be the best start to illuminate the path of research and social researcher can make your practice a good breath exercise.

However, it is worth considering a central element and which makes the difference regarding the mode and manner in which we face and read these new realities, the theory. The controversy about the role that must comply with the theory and relevance that will give it the researcher are very frequent. In this regard I must say that the theory is the element that is of importance of the research process. I.e., it is in the practice of research where it becomes operational theory and this is what gives meaning to the office of social research. This should translate into a sort of exercise "of hermeneutical phenomenology".

Key words: History; Method; Research; Hermeneutics.

\section{Generalidades}

El eje de orientación más evidente y que puede servir de punto de contraste viene a ser el hecho de que en el método histórico a los investigadores sociales les interesa seguir la pista al desarrollo en el tiempo y proceder a comparar estos procesos de desarrollo en las culturas. Aunque esta práctica no es una propiedad exclusiva del análisis histórico comparativo, sí deja ver la diferencia respecto a métodos de corte "positivo". Es decir, de alcance intermedio que se centran fundamentalmente en estudios anclados en un punto del tiempo y en un escenario específico.

Se revela así el rastro y la línea epistemológica de la tensión entre la compresión y la explicación, o la gran 
dicotomía de los paradigmas metodológicos casi opuestos; el positivismo y la hermenéutica. Los métodos históricos y sus fuentes de datos en las experiencias de la investigación social se han movido en estos ejes de convergencias y divergencias y "el curso de su definición va de la descripción a la explicación y/o interpretación. El gran problema de la decisión metodológica es la validación de la información y de los juicios de valoración" (Cáceres y González: 1994, p. 15).

Mostraré brevemente la ruta de esta tensión haciendo una reflexión de la investigación o estudio de localidades que tiene como eje orientador los procesos de larga duración y la experiencia histórica (Por ejemplo al mejor estilo de Orlando Fals Borda en sus cuatro volúmenes de Historia Doble de la Costa). En este tipo de experiencias, el método histórico se revela como anti-cronológico y no episódico de los relatos del pasado, de los héroes, los políticos y la clase dominante, la historia como método de investigación deberá estructurarse en el investigador como memoria colectiva, la cual, ha permitido configurar la identidad cultural. El método de la investigación histórica en estudio de localidades permite comprender los procesos o desarrollos por los que ha pasado una sociedad en un período determinado; es saber cuáles de estos procesos perduran en la actualidad como ejes de larga duración y cuales han cambiado. Evidentemente, la investigación histórica comparativa se revela como una técnica sumamente cualitativa. No obstante, acaso los analistas históricos no recurren con frecuencia a utilizar datos de series temporales con el propósito de controlar los cambios en las condiciones a lo largo del tiempo; con mayor razón en estudio de localidades, (datos sobre población, índices de delincuencia, de desempleo, morbilidad, censos, por ejemplo).

¿A qué se enfrentan entonces los investigadores con el método histórico, cuando sutilmente los tipos de análisis formal, estadístico y objetivo se atraviesan en la intención hermenéutica?

Para empezar, los datos disponibles que aparecen cuando se aplica el método histórico en la investigación social son infinitos, como infinitos son los datos cuantitativos que apoyan las investigaciones en trabajos de tipo histórico. Esta cualidad se sostiene por que los historiadores ya han aportado información sobre el tema del cual se está examinando. Un ejemplo válido es que para el estudio de la cuestión regional en el Departamento de Nariño, Colombia, se han encontrado cerca de 800 registros que aportan información sobre este tema, muchos de estos trabajos conjugan fuentes de tipo cualitativo con fuentes de tipo cuantitativo, entrar a analizar esta información constituye una buena base de información desde donde se puede emprender una investigación más precisa. Pero, ¿Qué me dicen estos registros sobre la confianza de su información con relación a lo que estoy indagando?, ¿Cuáles son algunos de los sesgos de los documentos y como ocuparse de ellos para corregirlos?

Surge aquí una orientación de orden metodológico en el tratamiento de 
las fuentes de datos, no se puede para el caso de la investigación histórica confiar en la precisión de los registros a no ser que:

Diversas fuentes apunten hacia la misma serie de "hechos", su confianza en ellos podría aumentar de forma razonable (...) Al mismo tiempo, siempre ha de recelar del sesgo en sus fuentes de datos. Si todos sus datos sobre el desarrollo de un movimiento político proceden del movimiento mismo, será difícil que consiga una visión completa de él (...) Cuando sea posible obtenga datos de distintas fuentes, de fuentes que representen diferentes puntos de vista. (Earl, 1996, p.452).

Estas dicotomías son propias de la naturaleza de los métodos de investigación. Sin embargo, ello previene al investigador sobre los sesgos si no conserva la claridad en la teoría, en el manejo de las fuentes de datos y el propio método de investigación. A mi juicio, el punto de partida es tener muy en cuenta que en el debate contemporáneo los paradigmas o programas de investigación social y la decisión y aplicación por los métodos históricos se distribuyen en dos posiciones polares; siguiendo a Cáceres y González (1994) estos son:

por un lado, la posición positivista u objetivista que considera los fenómenos sociales como cosas, es decir como fenómenos susceptibles de observación directa, de medición y de cuantificación estadística; y por otro lado, la posición interpretativa o hermenéutica, que considera los fenómenos sociales como formas simbólicas susceptibles de ser comprendidas e interpretadas (p. 55).

Lugares del campo del oficio del científico que revelan las tensionalidades metodológicas, gnoseológicas y epistemológicas que constituyen el objeto de la ciencia social y que han sido parte insoslayable de cada una de los paradigmas de la sociología, a saber:

- La tensión entre el sujeto y el objeto que implica, la pérdida de centralidad del sujeto para algunos y el redescubrimiento de los sujetos para otros.

- La tensión entre la acción social, las instituciones y las estructuras sociales.

- El recurrente problema del objetivismo y del subjetivismo.

- La tensión entre naturaleza y cultura.

- La relación entre individuo y sociedad.

- Los problemas del poder y del orden.

Volviendo ahora a los supuestos epistemológicos del método histórico, y como para dejar señalada una ruta de reflexión que se proyecte alivianar la tensión de estos paradigmas que tienen que ver con los métodos históricos y las fuentes de datos, traeré el comentario de Galindo sobre la propuesta de Thompson como caso ejemplar de resolución del conflicto de los paradigmas; tarea ésta considerada como una de las más audaces creaciones teóricas de los últimos tiempos por la bondad de conciliar los paradigmas de 
la investigación social que en principio se presentaban antagónicos.

Cáceres y González (1994) se refieren a Thompson así "Como cosa ejemplar de resolución del conflicto de paradigmas. Parece ser que lo objetivo y lo subjetivo no están reñidos y el asunto de la desnivelación en la composición y representación del mundo tiene salidas más que negociadas" (p. 18).

Ahora bien, quisiera terminar esta reflexión con algunos comentarios acerca del análisis de los datos históricos; claro, teniendo en cuenta que en la investigación histórica por tratarse de un método que privilegia básicamente lo cualitativo se presentan muchas dificultades para enumerar una serie de pasos a seguir en la tarea de analizar los datos históricos.

La pauta la da la herramienta para aproximarse del objeto de estudio, la experiencia en la investigación sociológica a través de la utilización de los métodos históricos la define el sociólogo alemán Max Weber quién utiliza el término "Verstehen" o comprensión cuando hace referencia a la cualidad de la investigación social. Para Weber es el investigador a través de la mente como se apropia de las circunstancias del objeto de estudio para captar adecuadamente por medio de la interpretación los actos de quienes ejecutan las acciones, los hechos, las intenciones y las voluntades. En la actualidad, los científicos sociales han dado en llamar a esta herramienta de análisis histórico bajo el nombre de "Hermenéutica" y a diferencia de los métodos que recurren a los datos cuantitativos apoyados en cálculos matemáticos, los resultados que se obtiene de la hermenéutica son más relativos, más difíciles de precisar y con más frecuencia se someten a debate.

Una de las razones para sustentar esta afirmación es que el investigador social en la dimensión histórica, debe buscar patrones de identificación de entre la inmensa variedad de datos y detalles con los que se topa en su investigación. Este ejercicio insoslayable de la experiencia del investigador equivale a lo que Weber desarrolló y dio en llamar como "Tipos Ideales" o modelos conceptuales compuestos de las características más significativas de los fenómenos sociales.

Así, por ejemplo, el propio Weber hizo una importante investigación sobre la burocracia. Habiendo observado diversas burocracias reales, Weber (1925) detalló aquellas cualidades esenciales de las burocracias en general; las áreas jurisdiccionales, la autoridad jerárquicamente estructurada, los archivos escritos, etc. Weber no se limitó a enumerar las características comunes de las burocracias reales que observó, sino que precisó una comprensión detallada de los elementos fundamentales del funcionamiento burocrático para crear un modelo teórico de burocracia perfecta (tipo ideal). (Earl, 1996, p.452).

Quiero terminar esta disertación declarando, como lo he intentado hacer en el desarrollo del texto, que la intención hermenéutica frecuente de los métodos históricos no se presenta, ni mucho menos se pretende presen- 
tarla como una posición metodológica excluyente respecto de la intención positivista que usualmente crítica. A este propósito nada más apropiado que la propia reflexión de Thompson que a continuación viene a manera de conclusión citada del texto de Cáceres y González (1994) expresando:

Existe, por supuesto, la tentación constante de tratar los fenómenos sociales, en general, y las formas simbólicas, en particular, como si fueran objetos naturales susceptibles de ser sometidos a diferentes tipos de análisis formal, estadístico u objetivo. Mi posición aquí no es la de considerar que tal tentación es totalmente desorientadora, que, por lo tanto, debe ser resistida a toda costa; ni la de considerar que el legado del positivismo debe ser erradicado de una vez por todas. Este punto de vista puede ser el de algunos proponentes radicales de lo que suele llamarse "enfoque interpretativo" en el análisis social, pero no es el mío. Mi razonamiento se encamina más bien a afirmar que los diferentes tipos de análisis formal, estadístico u objetivo son perfectamente apropiados y hasta de vital importancia en el análisis social, en general y en el análisis de las formas simbólicas, en particular; pero que, sin embargo, estos tipos de análisis deben ser considerados, a lo más, como enfoques parciales en el estudio de los fenómenos sociales y de las formas simbólicas. Son parciales porque, como nos lo recuerda la tradición hermenéutica, muchos fenómenos sociales son formas simbólicas y todas las formas simbólicas son constructos dotados de sentido que, por más exhaustivamente que se los someta al análisis formal u objetivo, suscitan inevitablemente problemas de comprensión e interpretación. Por consiguiente, los procesos de comprensión e interpretación tienen que ser considerados, no como una dimensión metodológica que excluya radicalmente el análisis formal u objetivo, sino más bien como una dimensión a la vez complementaria e indispensable respecto de la primera (p. 65).

Para recapitular con lo que viene dicho, el eje de orientación más evidente y que puede servir de punto de contraste viene a ser el hecho de que en el método histórico a los investigadores sociales les interesa seguir la pista al desarrollo en el tiempo, y proceder a comparar estos procesos de desarrollo en las culturas, fundamentalmente a partir de las siguientes dimensiones:

- Referentes de geografía física local.

- Información relacionada con la población.

- Actividades económicas.

- División política y administrativa.

- Información social y cultural.

- Información política.

Conviene para este propósito dar algunas pautas sobre la dirección gnoseológica en este tipo de práctica académica; sobre el contenido de la historia como método. Es decir, ¿Cómo opera el método histórico?

La historia no tiene la condición de recreación del pasado por el pasado, es más bien el conocimiento de los procesos o desarrollos históricos por los que ha pasado una sociedad, es saber cuáles de esos períodos perduran hoy como ejes de larga duración. 
Esta historia nos permite entender qué somos y que procesos nos han permitido que seamos así. A partir de este conocimiento podemos afianzar nuestra identidad y el conocimiento de lo propio.

La historia no es la versión positiva, cronológica y episódica de los relatos del pasado, de los héroes, los políticos, los gobernantes y la clase dominante, es en términos de Lucien Febvre y Marc Bloch, la nueva historia (1929 escuela de los anales). La historia se concibe como memoria colectiva en donde los hechos se entienden en cuanto se refieren a la vida del hombre en sociedad. "La ruta de lo histórico, no es lo oficial, es la ruta de la nueva historia que libera al investigador del riesgo que trae el navegar por las grandes superficies, lo global y lo homogéneo" (Salas, 2018, p. 15). La historia entendida así recrea territorialidades y estos dispositivos territoriales se refieren a una historia o están ligados a una en particular, a un pasado vivido en común por un grupo humano en un territorio, la historia de- fine los límites y una extensión que es espacio de producción y reproducción material. La región y las localidades entonces se sustentan por la memoria colectiva, por la percepción primaria, la noción de pertenencia y la diferenciación de otras regiones.

El método de la investigación histórica en estudios territoriales permite comprender los procesos o desarrollos por los que ha pasado una sociedad en un periodo determinado, nos permite saber cuáles de estos procesos perduran en la actualidad como ejes de larga duración y cuales han cambiado. Evidentemente, la investigación histórica comparativa así se revela como una técnica sumamente cualitativa y nos conduce a considerar la historia como método, en su dimensión propiamente heurística; más que un recetario rígido e inflexible, un abanico de posibilidades afianzado en el motor de la imaginación creadora de los investigadores $\mathrm{y}$ visible solo si aceptamos que hay muros epistemológicos que debemos derruir. 


\section{Referencias}

Cáceres, J. G., González, J. A. (1994). Metodología y cultura. Ediciones pensar la cultura: México.

De la Torre, N. (1999). Pensar la política. Publicado por la Universidad Autónoma de San Luis Potosí: México.

Earl, B. (1996). Manual para la práctica de la investigación social. Editorial Desclee de Brouwer: México.

Salas Salazar, V. F. (2018). Educación y Región. Pasto- Colombia: Editorial Universidad de Nariño. 